\title{
Processing and Characterization of Surface Treated Chicken Eggshell and Calcium Carbonate Particles Filled High-Density Polyethylene Composites
}

\author{
Radwan Dweiri ${ }^{a} *$ (D) \\ ${ }^{a}$ Al-Balqa Applied University, Faculty of Engineering, Department of Materials Engineering, 19117 \\ Al-Salt, Jordan
}

Received: February 4, 2021; Accepted: February 24, 2021

\begin{abstract}
The use of the biowaste chicken eggshell (ES) particles as an alternative filler for the inorganic calcium carbonate (CC) in polymeric materials was investigated in this study. ES and CC were chemically surface treated with stearic acid by dry and wet methods. Composites of ES and CC filled high-density polyethylene (HDPE) was prepared via melt mixing and their thermal-mechanical- and flow behavior was characterized. FTIR results showed some changes in the chemical structure of SA treated ES and CC. The mechanical properties of the composites fluctuated in their values based on the SA treatment method. The wet-treated HDPE/ES composites showed higher tensile and flexural strengths compared to that of the untreated ones which were also higher than their counterparts of HDPE/CC composites. The treated-ES particles motivated the crystallization process of HDPE compared to the untreated-ES and better flow properties was reported for composites with higher crystallinity and for HDPE/CC composites. Morphological observations showed particles agglomeration and void existence which led to deterioration of the mechanical properties.
\end{abstract}

Keywords: polymer composites, surface modification, chemical analysis, thermal analysis, mechanical testing, microscopy.

\section{Introduction}

The use of inorganic calcium carbonate (CC) as a filler is common in the production of polymer products. ${ }^{1}$ In recent years, there was also an interest in utilizing chicken eggshell (ES) as a bio-filler in polymeric materials and this was initially pronounced by Toro et al..$^{2-5}$ The high bio-calcium carbonate percentage of $95 \%$ in the ES composition revealed to the researchers to use it as a filler in polymeric materials products instead of using the common inorganic $\mathrm{CC}$ filler. ${ }^{6-8} \mathrm{~A}$ recent review conducted by Owuamanam and $\mathrm{Cree}^{9}$ to follow the progress of using the bio-filler ES waste in polymer composites has emphasized the importance of its use in the production of new thermoset and thermoplastic polymer composites. The polymer/ES similar to polymer/CC composites have been produced by different techniques such as: injection molding, extrusion/compression molding, film-casting, solution mixing, and hand-layup technique. ${ }^{10-14}$ In general, the use of such fillers either CC or ES has their advantages and disadvantages. The main advantage of using these fillers in compositing the polymeric materials is to reduce the cost by replacing a weight percentage of the relatively expensive polymeric materials with a cheaper filler material and filling load up to $40 \mathrm{wt}$.\% with ES or CC was reported in the literature. ${ }^{5,6,15-17}$ Moreover, these composites are more environmentally friendly. On the other hand, the degradation in the mechanical properties of neat polymeric materials when incorporating ES or CC was an important issue to be taken into consideration by the researchers. The values of tensile,

*e-mail: dr.dweiri@bau.edu.jo flexural, and impact strengths of these composites might be improved only at low filler loading, while their stiffness could be significantly improved with the increase in the filler content. Teixeira et al. ${ }^{18}$ showed that the addition of 2.5-4 $\mu \mathrm{m}$ untreated $\mathrm{CC}$ particles to HDPE provoked a significant decrease in its impact strength at 5 wt.\% CC and a slight decrease in the tensile strength compared to that of the neat HDPE. A comparison carried out by Azman et al. ${ }^{19}$ between the mechanical properties of HDPE/untreated ES and HDPE/untreated CC with 15 wt.\% filler content showed a similar trend of both types of composites in decreasing the tensile strength of about $23 \%$ compared to the pure HDPE. Their flexural strength also decreased but its value was higher for ES composites compared to $\mathrm{CC}$ ones which was attributed to higher tendency of agglomeration of the smaller $\mathrm{CC}$ particles. Khalaf $^{20}$ in his investigation on the mechanical properties of untreated CC filled HDPE observed that the tensile strength increased at 5 wt.\% CC by about $19 \%$ compared to pure HDPE and its value decreased, thereafter, by about $20 \%$ at $20 \mathrm{wt} . \%$ compared to that of the matrix. Wahab et al. ${ }^{21}$ showed a decrease in the impact strength of HDPE/untreated CC composites at all filling loads. Shuhadah and Supri ${ }^{14}$ showed a decrease of $50 \%$ in tensile strength of low-density polyethylene composites combined with 20 wt. $\%$ of $\mathrm{NaOH}$-treated ES of size $63 \mu \mathrm{m}$ while the tensile modulus increased by more than $100 \%$. Boronat et al. ${ }^{3}$ showed a decrease of $15 \%$ for composites of HDPE and $20 \mathrm{wt} \% \mathrm{ES}$ of size $25 \mu \mathrm{m}$ and a slight increase in the tensile and flexural modulus. The researchers attributed the decrease in the mechanical properties to the lack in 
adhesion bonding between the fillers and the matrix as well as the bad dispersion of the fillers. Therefore, previous studies in different directions have been carried out where some researchers have used nano-sized fillers to enhance the mechanical properties of the composites..$^{22}$ Due to the matrix hydrophobic nature and the fillers hydrophilic nature, other researchers have implemented different filler surface modification methods using different coupling agents to enhance the compatibility between the filler and the matrix as well as the filler dispersion in the matrix. ${ }^{23-26}$ Hydrogen bonding between the polymer and the filler which may be promoted by amine, carboxylic, and hydroxyl groups could improve the bonding between them. Inexpensive fatty acids such as stearic acid was also extensively used to improve the hydrophopocity of CC. ${ }^{27,28}$ Wet and dry coating methods of CC with a hydrophobic layer of SA was reported. ${ }^{19,29} \mathrm{ES}$ was also treated by stearic acid and observations of better dispersion and reduction in ES particles were reported by Shah et al. ${ }^{30}$ who also observed that a $22 \%$ increment in the elongation of epoxy/ES composites compared to neat epoxy and attributed that to the hydrogen bonding between the treated particles and matrix.

Finally, the motivation is still present among researchers to conduct further work in the processing of the polymer/ES composites and to better understanding their mechanical-thermal behavior as well as their other properties and compare them with that of the conventional mineral calcium carbonate $\mathrm{CC}$ composites. In this study, HDPE/ES and HDPE/CC composites were prepared via melt mixing and compression molding technique. The choice of HDPE as a matrix was due to that HDPE was and still one of the most consumed semicrystalline thermoplastics with increased demand market for many engineering applications and it is commonly reinforced with CC. HDPE composites were prepared at a filler concentration of $20 \mathrm{wt} . \%$ and it was reported in previous studies ${ }^{31}$ that CC content higher than this ratio resulted in highly deterioration in the mechanical properties of the composites with a more brittle-like failure manner. The surface treatment of $\mathrm{CC}$ particles with stearic acid which was suggested by the researchers in the literature ${ }^{32}$, using dry and wet methods, were also applied to ES particles in this study.

\section{Experimental}

\subsection{Materials}

Chicken eggshell (ES) was collected from the local market in Jordan and it was washed and dried at $80^{\circ} \mathrm{C}$ for overnight. The dried ES was then grinded into particles and sieved to obtain a size passed through 65-mesh and its density ranged between 2.5 and $2.6 \mathrm{~g} / \mathrm{cm}^{3}{ }^{24}$ Laboratory reagent calcium carbonate (CC) with a fine average particle size of less than $1 \mu \mathrm{m}$ was supplied by SDFCL-sd Fine Chem. Ltd., India. HDPE in granular form was supplied by $\mathrm{SABIC}^{\mathrm{R}}$, Saudi Arabia, with a density of $0.953 \mathrm{~g} / \mathrm{cm}^{3}$. Chemicals also provided for chemical treatment of ES and CC particles such as sodium hydroxide $(\mathrm{NaOH})$ solid which was supplied by Xilong Co., Ltd. (Guangdong, China) and Stearic acid in the form of flakes was purchased from Unichem Co., Ltd. (Mumbai, India).

\subsection{Chemical surface treatment of ES and CC particles}

A chemical surface treatment of ES and CC particles with SA was carried out by three different methods: (i) direct melt treatment method where ES or CC particles were manually dry mixed with SA before melt compounded with HDPE at $170{ }^{\circ} \mathrm{C}$ using two-roll mill; (ii) one-suspension method where a suspension of ES or $\mathrm{CC}, \mathrm{SA}$, and $\mathrm{NaOH}$ mixed with distilled water in a beaker and stirred for $10 \mathrm{~min}$ at $75^{\circ} \mathrm{C}$ and the mixture was then washed, filtered and oven-dried; (iii) two-suspension method where two separate suspensions (one suspension contained SA dissolved in $\mathrm{NaOH}$ at $75^{\circ} \mathrm{C}$ and the other suspension contained of ES or CC in distilled water at $75^{\circ} \mathrm{C}$ ) were mixed and stirred at $75^{\circ} \mathrm{C}$ for $15 \mathrm{~min}$ and then washed, filtered and oven-dried. Similar procedure for the last two methods was suggested by Cao et al. ${ }^{32}$ in their preparation and characterization of HDPE/CC composites.

\subsection{Preparation of HDPE/ES and HDPE/CC composites}

The composites of HDPE/ES or HDPE/CC were prepared by initially melting the HDPE granules using Brabender two-roll mill )C.W. Brabender Instruments, Inc. (for $2 \mathrm{~min}$ at $170{ }^{\circ} \mathrm{C}$ and a rotating speed of $50 \mathrm{rpm}$. The untreated or treated particles of ES or CC were then added to the molten HDPE and mixing process of all components was continued for another $8 \mathrm{~min}$. The resulting compounds were then hot pressed into square mold of $3 \mathrm{~mm}$ thickness at a temperature of $170{ }^{\circ} \mathrm{C}$ and a pressure of $15 \mathrm{KPa}$ for $10 \mathrm{~min}$. Samples for impact, tensile, and flexural tests were prepared according to different ASTM standards. Eight types of composites were prepared with 80 to 20 weight ratio of HDPE to ES or HDPE to CC and compared with the neat HDPE and they were given the designation shown in Table 1.

\subsection{Characterization of composites}

A Fourier transform infrared (FTIR) spectrophotometer (model- Burker Tensor 27 SN_3880, Germany) was utilized to identify the functional groups. $\mathrm{KBr}$ is used as a carrier for the sample in IR spectrum scanned from 400 to $4000 \mathrm{~cm}^{-1}$ with a resolution of $4 \mathrm{~cm}^{-1}$.A differential scanning calorimeter (DSC, Perkin Elmer7) was used to investigate the thermal behavior of the composites and it carried out using oxygen purging at a rate of $50 \mathrm{~mL} / \mathrm{min}$. Aluminum pans containing 6 to $7 \mathrm{mg}$ of composite material were used and the specimens

Table 1. Designation of HDPE/ES and HDPE/CC composites.

\begin{tabular}{llc}
\hline \multicolumn{1}{c}{ Sample Type } & Designation & $\begin{array}{c}\text { Filler-to-PP } \\
\text { weight ratio } \\
\text { (wt.\%) }\end{array}$ \\
\hline HDPE/ES (untreated) & HDPE/UES & $20: 80$ \\
\hline HDPE/ES (direct melt mixing) & HDPE/TES1 & $20: 80$ \\
\hline HDPE/ES (one-suspension) & HDPE/TES2 & $20: 80$ \\
\hline HDPE/ES (two-suspensions) & HDPE/TES3 & $20: 80$ \\
\hline HDPE/CC (untreated) & HDPE/UCC & $20: 80$ \\
\hline HDPE/CC (direct melt mixing) & HDPE/TCC1 & $20: 80$ \\
\hline HDPE/CC (one-suspension) & HDPE/TCC2 & $20: 80$ \\
\hline
\end{tabular}


were heated to $200^{\circ} \mathrm{C}$, and then cooled to room temperature at a heating and cooling rates of $10^{\circ} \mathrm{C} / \mathrm{min}$. Tensile and flexural test of the composites were carried out according ASTM D638 and ASTM D790 standard respectively using universal testing machine (HOYTOM, Model DI-922 FL, Spain) at 2 and $1 \mathrm{~mm} / \mathrm{min}$ using a load-cell with the capacity of $50 \mathrm{kN}$. Rectangular specimens were prepared of dimensions $(165 \times 13 \times 3.2 \mathrm{~mm}) \mathrm{mm}$ for tensile test and of dimensions $(50.8 \times 12.7 \times 3.2 \mathrm{~mm})$ for flexural test. The Izod notched impact test was also carried out according to ASTM D265 standard using a Ceast pendulum impact tester and rectangular specimens $(64 \times 12.7 \times 3.2 \mathrm{~mm})$. The melt flow index $(\mathrm{MFI})$ of the composites was determined according to ISO1133 using Ceast melt flow tester measured at $190{ }^{\circ} \mathrm{C} / 2.16 \mathrm{~kg}$. Finally, the fractured surface of the composites and the distribution of particles throughout the matrix were examined by scanning electron microscopy (FEI Quanta 600 FEG-SEM). The specimens were coated with Platinum/Palladium Pt/Pd. The average value of three specimens of each type was considered in the mechanical testing.

\section{Results and Discussion}

\subsection{FTIR results}

The results of FTIR analysis for ES, CC, HDPE, HDPE/ES, and HDPE/CC are shown in Figures 1 and 2. The FTIR spectra of the untreated ES (Figure 1a) showed three characteristic peaks located at 1421,875 , and $687 \mathrm{~cm}^{-1}$, which represented the stretch and bending vibrations of $\mathrm{CO}_{3}{ }^{2-}$ ion. ${ }^{30}$ Between 3000 and $2500 \mathrm{~cm}^{-1}$, the absorption bands were identified as organic matter (C-H stretching) and an absorption band at $3421 \mathrm{~cm}^{-1}$ was related to the stretching absorption of $\mathrm{H}_{2} \mathrm{O}$. Similar characteristics to ES structure were observed for $\mathrm{CC}$ from FTIR spectra shown in Figure 1b at slightly different positions for the peaks. FTIR spectra of SA in Figure 1a and $1 \mathrm{~b}$ showed adsorption peaks at about 2921 and $2843 \mathrm{~cm}^{-1}$ which are attributed to stretching vibrations of $-\mathrm{CH}_{2}$ groups ${ }^{33}$ In the low-frequency region, the peak at $1703 \mathrm{~cm}^{-1}$ was attributed to carbonyl $(-\mathrm{COOH})$ stretch and there were different peaks located in the range 500 to $1500 \mathrm{~cm}^{-1}$. In general, after surface treatment the particles with $\mathrm{SA}$, a chemical reaction of SA with ES or with CC and a formation of a hydrophobic monolayer film might be expected and this can be noticed in FTIR analysis usually by the appearance of new absorption peaks or the absence of old ones, a change in their intensities, and a broadening in peaks or shifting in their positions. The surface treatment of ES with SA resulted in the appearance of new characteristic peaks of at 1578, 1539, and $1468 \mathrm{~cm}^{-1}$ for one-suspension treated ES and at 1585 and $1539 \mathrm{~cm}^{-1}$ for two-suspension treated ES indicating the reaction of $\mathrm{CO}_{3}{ }^{2-}$ existed on the surface of ES particles with SA. ${ }^{34}$ On the other hand, the increase of the intensities of $\mathrm{C}-\mathrm{H}$ vibration bands between 3000 and 2500 indicated that the surface of ES was coated by SA, so that the hydroxyl number was reduced. ${ }^{35}$ The peaks identified as organic matter stretching increased in treated ES which may point out to a chemical reaction of SA with ES particles. ${ }^{36}$

The chemical structure of HDPE and HDPE/ES and HDPE/CC was examined by FTIR as shown in Figure 2. A stretching vibrations of $-\mathrm{CH}_{3}$ appeared at a frequency range

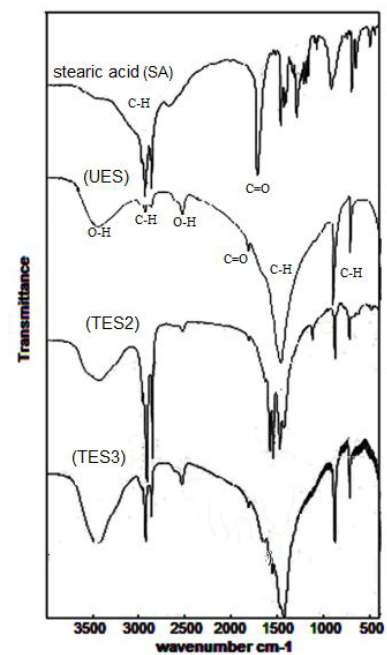

(a) ES

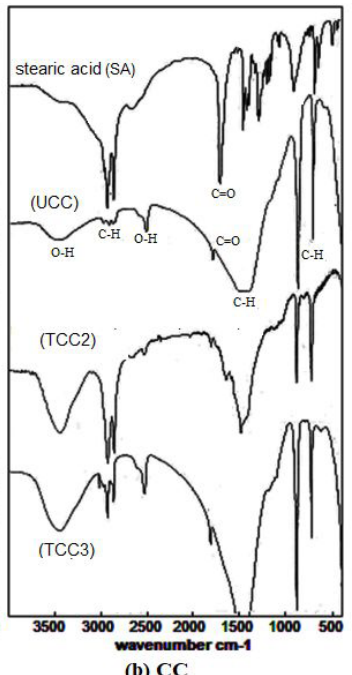

(b) $\mathrm{CC}$
Figure 1. FTIR results of untreated and SA treated (a) ES and (b) CC.

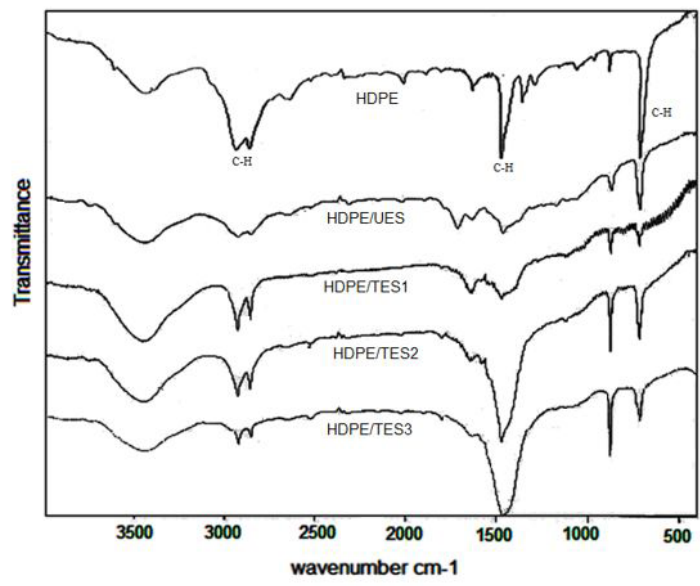

(a) HDPE/ES composites

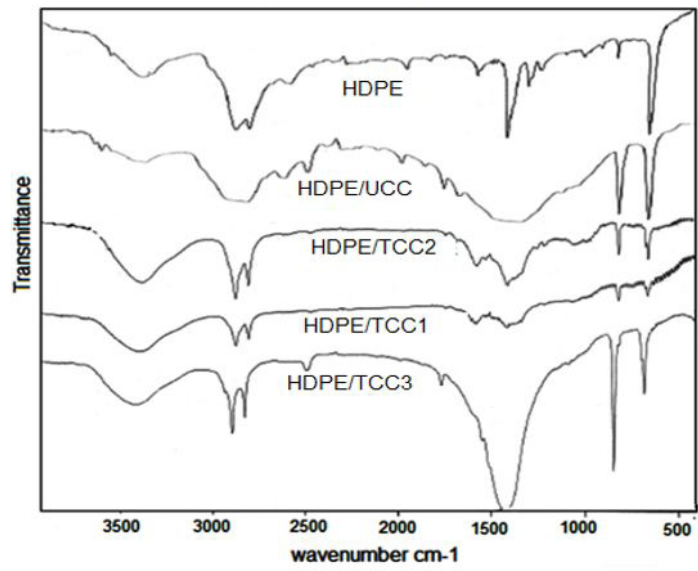

(b) HDPE/CC composites

Figure 2. FTIR results of untreated and treated (a) HDPE/ES and (b) HDPE/CC composites.

of 2921-2843 $\mathrm{cm}^{-1}$, a bending and a rocking vibration of $-\mathrm{CH}_{2}$ appeared at $1468 \mathrm{~cm}^{-1}$ and at $703 \mathrm{~cm}^{-1}$ respectively corresponded to the native bonds present in HDPE. ${ }^{37}$ The weak absorption 
band at 1078 and $875 \mathrm{~cm}^{-1}$ can be assigned to the vinyl groups according to Apone et al. ${ }^{38}$. There is also small absorption bands appeared in the range $1625-2000 \mathrm{~cm}^{-1}$ which related to carbonyl groups appearance. According to the FTIR spectra, all the peaks of the HDPE/ES and HDPE/CC composites are in conformity with those of HDPE. This indicates that the main chemical structure of the composites is HDPE and it is an indication to bad compatibility. Azman et al. ${ }^{19}$ reported that HDPE composites showed, to some extent, similar spectra in the presence of ES or CC. Basilia et al. ${ }^{39}$ reported that the changes in the transmittance shown on FTIR of PE, PP, and PVC by the addition of CC particles into them indicating that there was a direct binding between the fillers and matrix.

\subsection{DSC and thermal properties}

The DSC heating and cooling curves of untreated and treated HDPE/ES and HDPE/CC composites are shown in Figure 3 and the thermal parameters obtained from DSC measurements are tabulated in Table 2 . The crystallinity percentage $\left(\mathrm{X}_{\mathrm{c}}\right)$ was calculated according to following equation: ${ }^{32}$

$$
\mathrm{X}_{\mathrm{c}}=\frac{\Delta \mathrm{H}_{\mathrm{m}}}{\mathrm{w}_{\mathrm{p}} \times \Delta \mathrm{H}_{100}} \times 100 \%
$$

The $\Delta \mathrm{H}_{\mathrm{m}}$ is the melting heat in $\mathrm{J} / \mathrm{g}, \Delta \mathrm{H}_{100}$ is the melting heat for $100 \%$ crystalline HDPE which is equal to $293.6 \mathrm{~J} / \mathrm{g}$ according to Cao et al. ${ }^{32}$, and $\mathrm{w}_{\mathrm{p}}$ is the weight fraction of HDPE in the sample. As can be observed from Table 1, the melting temperature $\left(\mathrm{T}_{\mathrm{m}}\right)$ decreased by 3 to $7^{\circ} \mathrm{C}$ for the composites compared to that of the neat HDPE, while there was a slight increment by up to $2{ }^{\circ} \mathrm{C}$ in the recrystallisation temperature $\left(\mathrm{T}_{\mathrm{c}}\right)$ of the composites. The decrease in $\mathrm{T}_{\mathrm{m}}$ was also observed by Cao et al. ${ }^{32}$ and due to the resulted thinner lamellar crystallites in the composites compared to that of the pure HDPE. Bartczak et al. ${ }^{40}$ observed in a previous study an increase in $\mathrm{T}_{c}$ up to $1^{\circ} \mathrm{C}$ or lower which was dependent on the particle size and indicated to some effect on nucleation of HDPE crystallites. The significant decrease in the melting heat $\left(\Delta \mathrm{H}_{\mathrm{m}}\right)$ and the degree of crystallinity of the composites compared to that of the neat HDPE indicated a lower nucleating ability of the particles. This was more pronounced in case of HDPE/ES composites. The decrease in $\Delta \mathrm{H}_{\mathrm{m}}$ clearly decreased the amount of energy required to break the solid for of the HDPE. It was reported by Lazzeri et al. ${ }^{41}$ that untreated CC particles as well the SA treaded ones have a small nucleating effect on HDPE which can be observed in this study by the small fluctuation of the crystallinity values of HDPE/CC composites about that of the neat HDPE. The improvement in both $\Delta \mathrm{H}_{\mathrm{m}}$ and $\mathrm{X}_{\mathrm{c}}$ was clear in treated HDPE/ES composites compared to that of untreated ones induced a higher heterogeneous nucleating and a higher crystallinity of HDPE. The HDPE/ES composites treated by two-suspensions method showed slightly better results than the ones treated by one-suspension method and significantly better results than direct-melting treated composites. Similar trend was observed for HDPE/CC composites regarding to melting heat but a slight increase in the degree crystallinity of treated composites was found compared to that of untreated

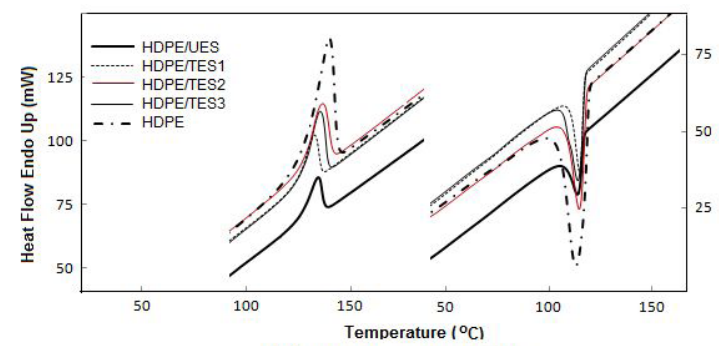

(a) HDPE/ES composites

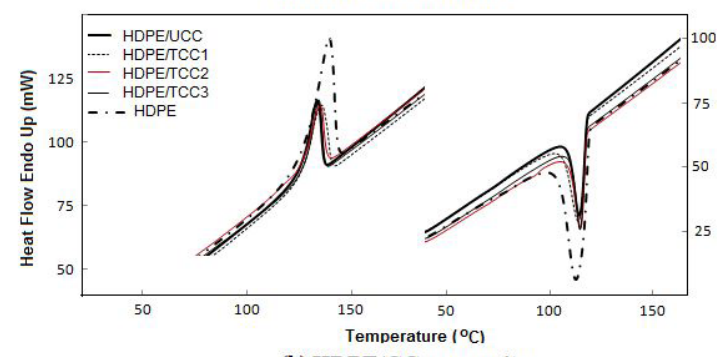

(b) HDPE/CC composites

Figure 3. DSC heating and cooling curves of (a) HDPE/ES composites and (b) HDPE/CC composites.

Table 2. DSC thermal parameters of HDPE, HDPE/ES composites, and HDPE/CC. composites.

\begin{tabular}{lccccc}
\hline & $\begin{array}{c}\mathrm{T}_{\mathrm{m}} \\
\left({ }^{\circ} \mathrm{C}\right)\end{array}$ & $\begin{array}{c}\Delta \mathrm{H}_{\mathrm{m}} \\
(\mathrm{J} / \mathrm{g})\end{array}$ & $\begin{array}{c}\mathrm{T}_{\mathrm{c}} \\
\left({ }^{\circ} \mathrm{C}\right)\end{array}$ & $\begin{array}{c}\Delta \mathrm{H}_{\mathrm{c}} \\
(\mathrm{J} / \mathrm{g})\end{array}$ & \%crystallinty \\
\hline HDPE & 139.3 & 179.1 & 113.6 & 167.5 & 61.0 \\
\hline HDPE/UES & 133.6 & 112.7 & 114.6 & 128.1 & 48.0 \\
\hline HDPE/TES1 & 132.3 & 119.1 & 115.3 & 132.8 & 50.7 \\
\hline HDPE/TES2 & 136.0 & 128.3 & 115.3 & 140.7 & 54.6 \\
\hline HDPE/TES3 & 135.0 & 133.7 & 114.6 & 139.0 & 56.9 \\
\hline HDPE/UCC & 133.6 & 134.4 & 114.9 & 152.0 & 57.2 \\
\hline HDPE/TCC1 & 135.3 & 148.3 & 114.9 & 153.7 & 63.1 \\
\hline HDPE/TCC2 & 133.6 & 141.4 & 115.6 & 152.2 & 60.2 \\
\hline
\end{tabular}

composites and neat HDPE. Saeedi and Sharahi ${ }^{42}$ reported that incorporating CC particles played a role in the arrangement of the chain molecules of HDPE and acting as nucleating agent which makes HDPE crystallize heterogeneously. Other researchers $^{32}$ found that the excess or insufficient surfactant of such particles diminished the nucleating ability. Higher $\Delta \mathrm{H}_{\mathrm{m}}$ and $\mathrm{X}_{\mathrm{c}}$ indicated a better surfactant of ES and CC. The change in the crystallinity was only supported by a slight change in the recrystallization temperature $\left(\mathrm{T}_{\mathrm{c}}\right)$. The increase in $\mathrm{T}_{\mathrm{c}}$ meant the acceleration of crystallization during cooling.

\subsection{Melt flow index}

MFI plays a curial role in controlling the processability of HDPE composites. ${ }^{43-45}$ The melt flow of the roll-milled HDPE and its composites were tested and the results are shown in Figure 4. It was observed that there was a decrease in the MFI by the addition of the $20 \mathrm{wt} . \%$ UES to HDPE, while no significant change occurred on MFI by the addition of UCC compared to that of the pure HDPE. Sahai et al. ${ }^{46}$ reported that the decrease in MFI is attributed to the agglomeration of the particles which results in restriction of flow for untreated $\mathrm{CC}$ filled polyphenylene oxide composites. They also found 

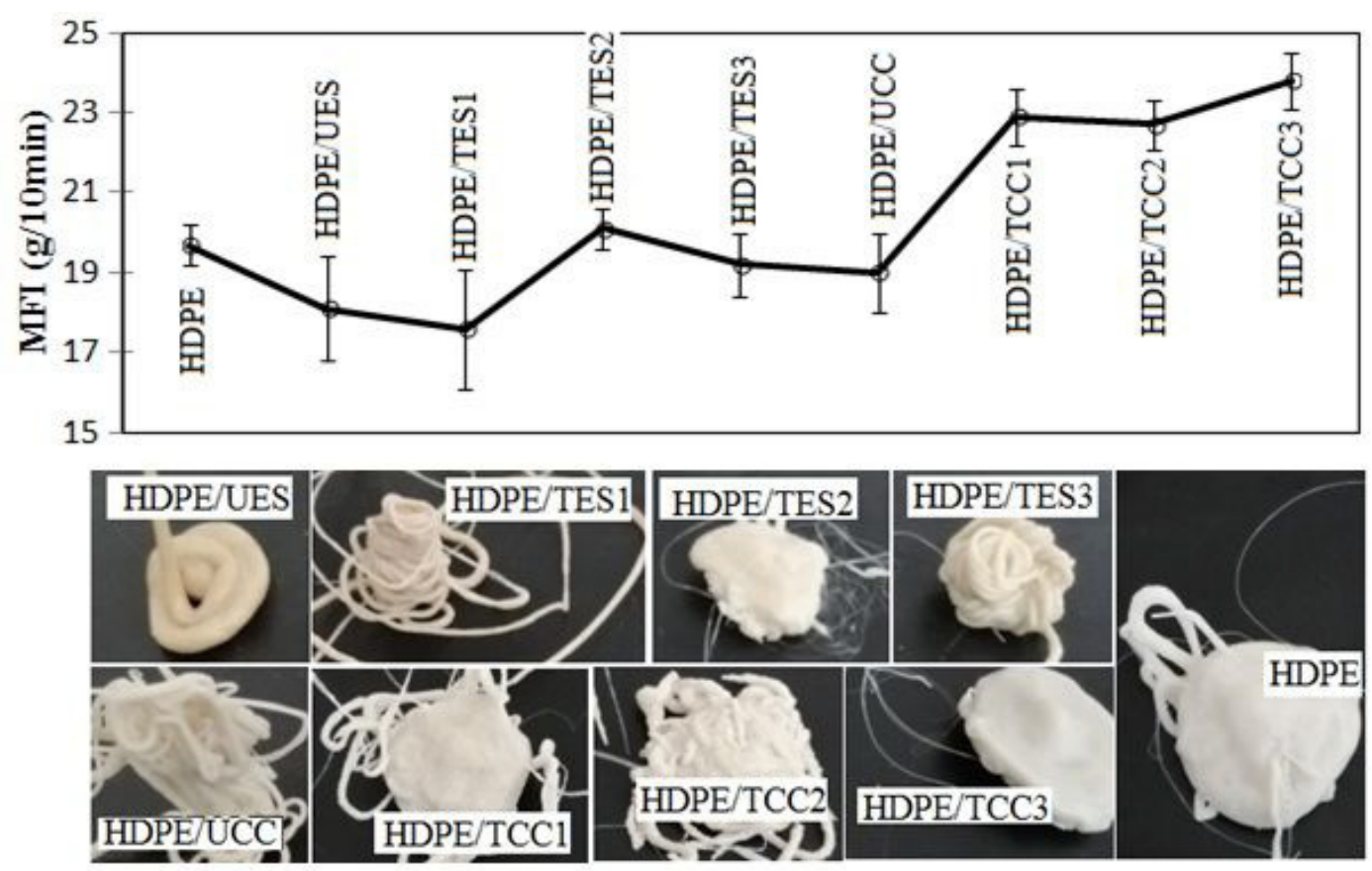

Figure 4. The values of MFI and the images of extrudates of the HDPE and its composites.

that the treated CC composites improved adhesion between the constituents and lowered the agglomeration but the good adhesion also contributed to reduce the MFI at a filler content less than $10 \mathrm{wt} . \%$. Jang ${ }^{47}$ in his study of polycarbonate (PC) composites explained the reduction of MFI by the large size of talc particles which act as flaws and their tendency to slide due to the flatly shape specially at higher concentrations. In Figure 4, the treated composites showed an improved melt flow properties compared to that of the untreated ones, especially for HDPE/CC composites. Adeosun et al. ${ }^{48}$ indicated that $\mathrm{CC}$ is capable to improve the processability and the plasticity of the polymer. Apone et al..$^{38}$ claimed that chain scissions and chain branching occurred during the degradation of HDPE control the MFI and its value, on average, increases or decreases based on the predominant factor. Ertürk and Şahin ${ }^{43}$ found that the addition of $\mathrm{CC}$ up to $20 \mathrm{wt} \%$ to HDPE has no influence on MFI, while higher filler loadings increased MFI of the composites.

\subsection{Mechanical properties}

The investigation of the mechanical properties of the composites is important where any improvement occurs in them reflects the ability of the matrix to transfer stress to the filler which depends on the adhesion properties, particle size, and filler loading ${ }^{49}$ The tensile, impact, and flexural strength for HDPE and its composites are shown in in Figures 5 and 6. A reduction of about $40 \%$ was observed in the value of the tensile strength as HDPE filled by $20 \mathrm{wt} . \%$ untreated ES. This deterioration in tensile strength was expected and reported by many researchers, e.g. Shuhadah and Supri ${ }^{14}$ reported a reduction of $50 \%$ in the tensile strength of low density polyethylene (LDPE) filled with $20 \mathrm{wt} . \%$ of untreated ES having a particle size of $63 \mu \mathrm{m}$. Hayeemasae et al. ${ }^{50}$ reported a $44 \%$ reduction in the tensile strength of polystyrene (PS) $/ 20 \mathrm{wt} . \%$ untreated ES composites with ES particle size less than

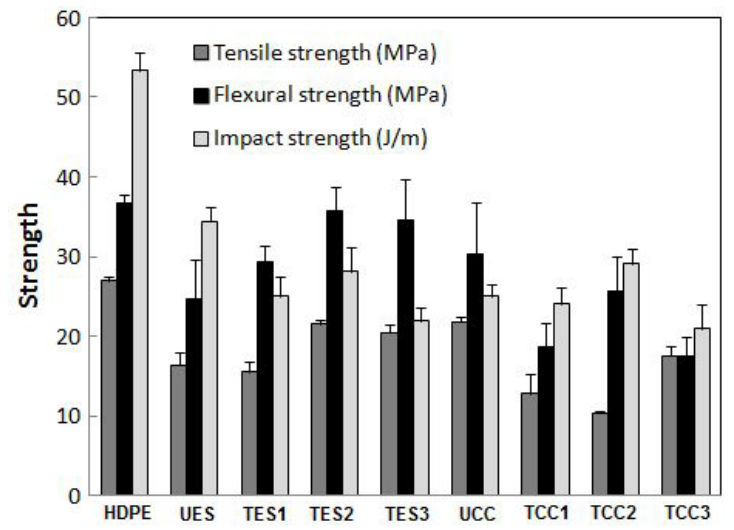

Figure 5. Mechanical strengths of HDPE, HDPE/ES composites, and HDPE/CC composites.

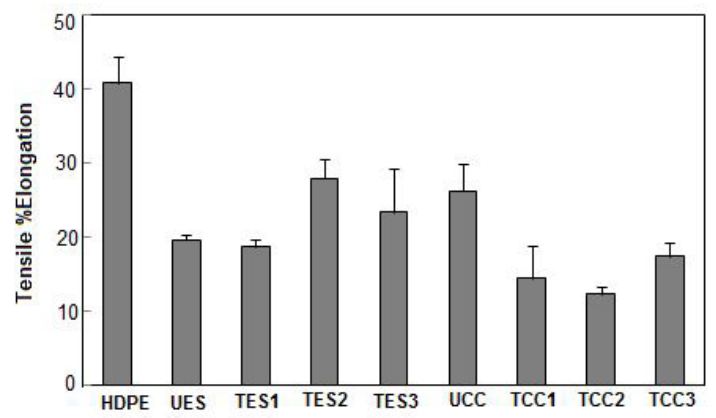

Figure 6. Tensile \%Elongation of HDPE and its composites.

$75 \mu \mathrm{m}$ and Murugan et al. ${ }^{23}$ reported a decrease of $18 \%$ for HDPE $/ 22.5$ wt. $\%$ ES ( $7 \mu \mathrm{m}$ particle size). They attributed the decrease in tensile strength to the poor interfacial adhesion between the components as well as the agglomeration of the 
particles. But compared to untreated HDPE/ES composites in this study, a clear improvement of about $25 \%$ occurred in the value of tensile strength of treated ES by one- or two-suspension methods (HDPE/TES2 and HDPE/TES3). Cao et al. ${ }^{32}$ found that the wet treated processes was more effective in improving the tensile strength due to the better interfacial adhesion between the filler and the matrix which could be observed from morphological changes shown in section 3.5. The treated HDPE/CC composites showed no improvement in tensile strength compared to untreated ones and some improvement was noticed for two-suspension HDPE/TES3 composites compared the other treated ones. Mohan et al. ${ }^{22}$ attributed the increase and decrease in the tensile strength of nano-EG filled epoxy composites to the action of the particles or agglomerates as a barrier to the crack propagation resulting in an increased strength and, on the other side, the action of particles or agglomerates as a stress concentration which aid the crack propagation and reduce strength. According to the flexural strength, the treated HDPE/ES composites showed a strength value near to that of neat HDPE and higher than that of untreated ones. The increase in the crystallinity of treated HDPE/ES composites compared to untreated ones may have a positive impact on the increase in their flexural strength values. Melo et al. ${ }^{51}$ in their study of the mechanical properties of titanate modified mollusk shell-waste filled HDPE composites pointed out to the enhancement in the flexural strength of the composites by their higher crystallinity compared to the neat HDPE which might overcome the negative impact of the particle agglomeration. The trend of flexural strength for HDPE/CC composites is similar to that of tensile strength except and increase of the strength value was observed for treated HDPE/CC composites by one-suspension compared to other types of treated ones. Regarding to the impact properties of the composites which is shown in Figure 5, a decrease in the impact strength of $\sim 35 \%$ and $54 \%$ was reported for HDPE/UES and HDPE/UCC respectively. Similar trend was reported by Buakaew ${ }^{52}$ in his investigations of the mechanical properties of HDPE/ES composites who found that the impact strength of these composites at $20 \mathrm{wt} . \%$ ES reduced by $\sim 55 \%$ compared to the neat HDPE and the presence of particles had negative impact on the ability of composite to absorb energy before rupture. He also found that the smaller the particle size the higher the impact strength due to lower the voids content which act as stress concentrator. Bonse and Molina ${ }^{53}$ found that the impact strength of polyamide $6 / 20$ wt. $\%$ CC composites decreased by $\sim 50 \%$ compared to the neat matrix regardless the size and the type of the $\mathrm{CC}$ filles. It was observed from the impact results in Figure 5 that there was no clear improvement in the impact strength appeared for treated composites compared to untreated ones in all types of composites. The impact strength for treated ES composites was almost similar to their counterparts of treated CC composites. The ductility results of composites are shown in Figure 6 and it was noticed that it decreased for HDPE by incorporating either ES or CC but it could be observed some increment in the ductility of treated HDPE/ES composites by one- and two-suspension method compared to that of untreated or direct-melting ones. Murugan et al. ${ }^{23}$ reported that the addition of untreated ES into HDPE reduced its ductility by $\sim 16 \%$ at 20 wt. $\%$ filler content and by $~ 30 \%$ for silane treated ES/HDPE composites compared to that of neat matrix. They attributed the reduction in the ductility by that the fillers usually restrain the movement of polymer chains during tension resulting in a strain localization which leads to a stiffer and a more brittle material. ${ }^{54,55}$ While the further decrease in the ductility of silane treated ES/ HDPE was related to the better adhesion which requires higher stress for small deformation. Shah et al. ${ }^{30}$ reported a reduction of $25 \%$ in the ductility of epoxy $/ 20 \mathrm{w} . \%$ UES and a rise of $\sim 22 \%$ for SA treated ES composites at same filler content and the explained the improvement in the ductility by the lower protein content in ES, and more carbonyl groups in TES and the better dispersion of the particles, as well as the reduction in the crystal size and introducing ore hydrogen bonding. The ductility of HDPE/CC composites reduced further for treated composites compared to that of untreated ones as shown in Figure 6.

\subsection{Morphological observations}

The dispersion of ES and CC particles and their compatibility with the matrix were investigated from the tensile fracture surface analysis of the specimens and their SEM micrographs are shown in Figures 7 and 8 at four different magnifications for each type of composites. The ES particles appeared to have a combination of fine and coarse plate-like and sphere-like irregular morphologies while the CC particles had finer regular sphere-like morphologies. The SEM images of HDPE/UES showed a more flattened shaped particles which were not or partially wetted with the matrix and some agglomerates were found. The bad adhesion evidenced by the clear and smooth surfaces of the particles and their detachment during fracture. The agglomeration of the particles might contribute to the formation of some voids and the porosity appearance led to increase their content on the surfaces. The fracture surface of HDPE/UCC composites appeared with smaller particle size and higher surface area and slightly better embedded particles in the matrix which reflected a higher tensile strength and more ductility compared to HDPE/UES composites, but agglomerates and voids still existed. On the other hand, preparing the composites by directly adding the dry SA and filler particles to the molten HDPE showed no improvement in the particles dispersion and adhesion with higher voids and porosity appearance which resulted in a further deterioration in the mechanical properties compared to untreated composites. Regarding to the wet treated HDPE/ES and HDPE/CC composites, the particles agglomeration and the void existence were also found. The wet treated ES particles were better embedded in the matrix and this was more pronounced in the case of two-suspension treated method. Moreover, the reducing in the treated ES particle size and the more their spherical morphology contributes to improve mechanical properties of the composites. Owuamanam ${ }^{24}$ found that the SA treated ES had a narrower particle size distribution compared to untreated particles and reduced the agglomerate size which might be due to the removal of bioorganic impurities. Better continuity of the matrix phase is also shown for two-suspension treated HDPE/TES3 and HDPE/TCC3 composites. More void contents and pulled out particles were noticed in HDPE/TCC2 composites. The plate-like shape of ES particles allowed better interfacial adhesion with the matrix at their edges compared to sphere-like particles. 


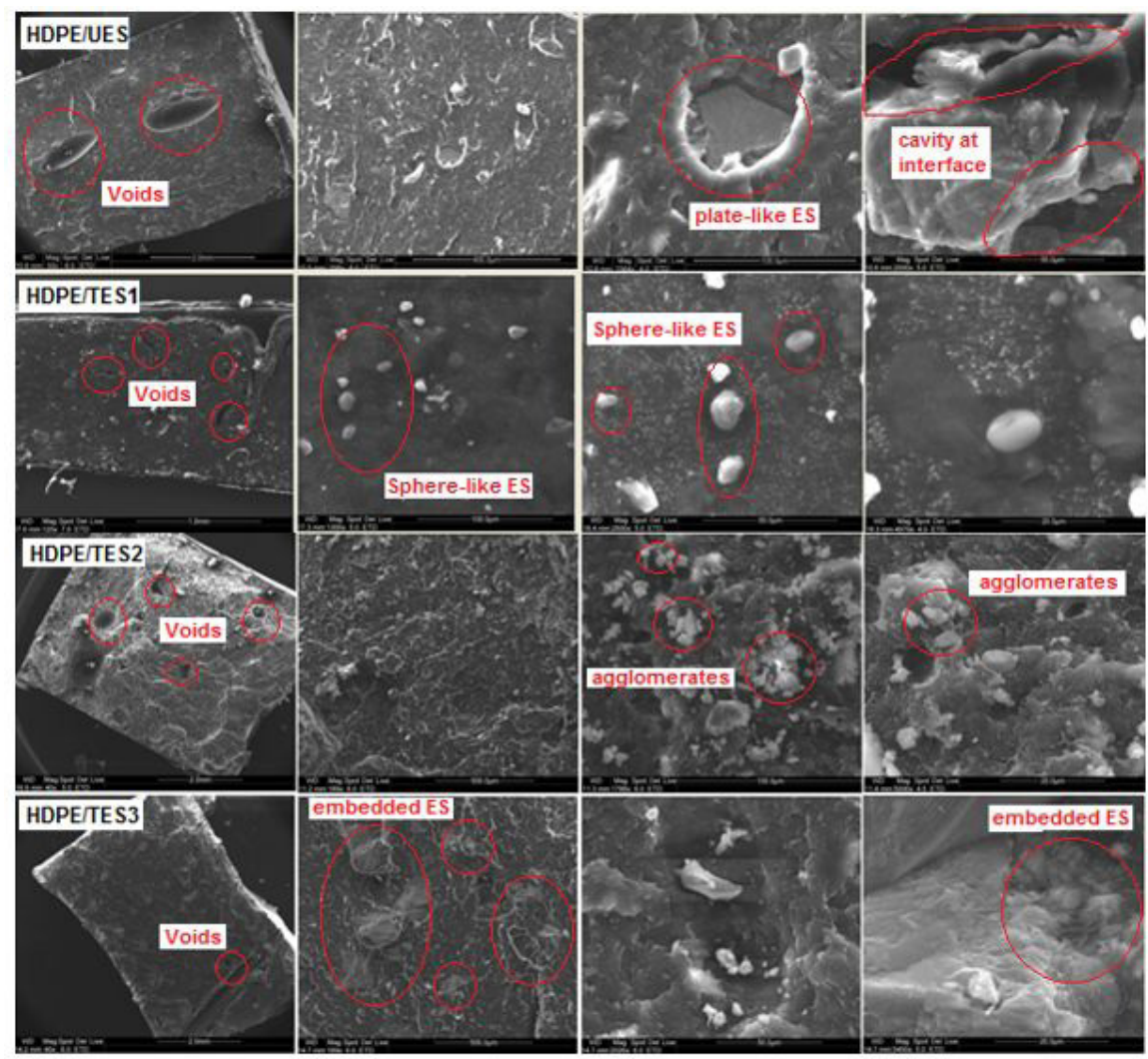

Figure 7. SEM images of tensile fracture surface of HDPE/ES composites.

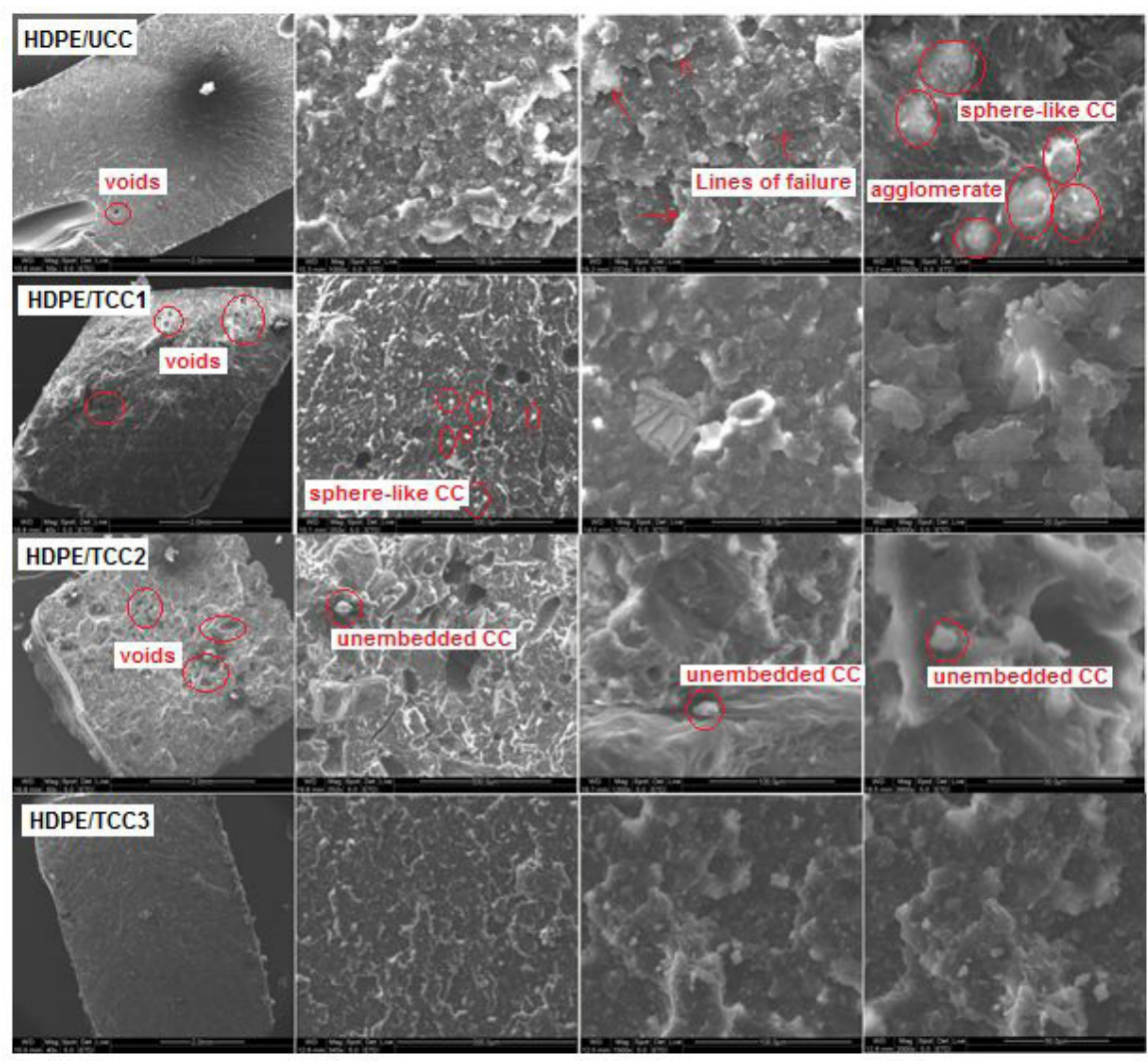

Figure 8. SEM images of tensile surface fracture of HDPE/CC composites. 
It is also known that the finer the particles the more sever is the problem of its dispersion in the matrix such as in case of HDPE/CC composites. On the other hand, Murugan et al. ${ }^{56}$ showed that the lower surface area of the large ES particles leads to their agglomeration. The rough surface topology of the composites indicated that they still ruptured in a ductile manner which might be due to more crack initiation and propagation sites.

\section{Conclusion}

In this study, the influence of the addition of ES as a filler to HDPE and their effect on its thermal, melt flow, and mechanical properties was investigated and compared to the conventional HDPE/CC composites. The chemical surface treatment of the particles with SA was also investigated for both types of fillers and their composites and the FTIR results showed some chemical structural changes of the ES and $\mathrm{CC}$ particles after the acidic treatment indicating the reaction of the SA with the particles. It is worth to mention that an optimization process of the concentration of SA, temperature, and time is needed in the future to further investigate the formation of a sufficient coating monolayer of SA on ES or CC surface. The change in the thermal properties of the neat HDPE occurred by incorporating ES or CC particles and the nucleating ability of ES or CC was investigated and the treated composites showed higher crystallinity values compared to the untreated ones mainly for HDPE/ES composites. The crystallinity and the flow properties of HDPE/CC composites were better enhanced compared to HDPE/ES composites. A Slight change occurred in the melting points of the composites. The improvement in the mechanical properties of treated HDPE/ES composites was clear compared to that of untreated ones and it was more pronounced than that of HDPE/CC composites. This motivates us to use the ES powder as an alternative to $\mathrm{CC}$ in plastics products. From the morphological observations it was found that some of the treated particles are partially or completely embedded in the matrix and others were not wetted with the matrix which might related to the amount and homogeneity of the coating of the particles with SA. The existence of some voids and particles agglomeration might also be related to the processing technique besides the adhesion characteristics. The use of similar particle size and shape of $\mathrm{ES}$ and $\mathrm{CC}$ for the purpose of comparison, the combination of both fillers at once in the matrix, and the adoption of more efficient processing technique are also some recommended work for future studies in this area.

\section{Acknowledgment}

The author acknowledges the support of Al-Balqa Applied University in offering the materials and facilities for this study. Special thanks to Eng. Ala'a Al-ajarmeh in Dar AlDawa Plant for their help in conducting FTIR analysis for the samples.

\section{References}

1. Rothon R. Fillers for Polymer Applications. Cham, Switzerland: Springer International Publishing; 2017. (Polymers and Polymeric Composites: A Reference Series).
2. Intharapat P, Kongnoo A, Kateungngan K. The potential of chicken eggshell waste as a bio-filler filled epoxidized natural rubber (ENR) composite and its properties. J Polym Environ. 2013;21:245-58.

3. Boronat T, Fombuena V, Garcia-Sanoguera D, SanchezNacher L, Balart R. Development of a biocomposite based on green polyethylene biopolymer and eggshell. Mater Des. 2015;68:177-85.

4. Hassan SB, Aigbodion VS, Patrick SN. Development of Polyester/ Eggshell Particulate Composites. Tribol. Ind. 2012;34(4):217-25.

5. Toro P, Quijada R, Yazdani-Pedram M, et al. Eggshell, a new bio-filler for polypropylene composites. Mater Lett. 2007;61:4347-50

6. Ghabeer T, Dweiri R, Al-Khateeb S. Thermal and mechanical characterization of polypropylene/eggshell biocomposites. J Reinf Plast Compos. 2013;32(6):402-9.

7. Supri AG, Ismail H, Shuhadah S. Effect of polyethylene-grafted maleic anhydride (PE-g-MAH) on properties of low density polyethylene/eggshell powder (LDPE/ESP) composites. Polym Plast Technol Eng. 2010;49:347-53.

8. Iyer KA, Torkelson JM. Green composites of polypropylene and eggshell: effective biofiller size reduction and dispersion by single-step processing with solid-state shear pulverization. Compos Sci Technol. 2014;102:152-60.

9. Owuamanam S, Cree D. Progress of bio-calcium carbonate waste Eggshell and Seashell Fillers in Polymer Composites: A Review. Journal of Composites Science. 2020;4(70):1-23.

10. Lin Z, Zhang Z, Mai K. Preparation and properties of eggshell/ $\beta$ polypropylene bio-composites. J Appl Polym Sci. 2012;125:61-6.

11. Feng Y, Ashok B, Madhukar K, Zhang J, Zhang J, Reddy $\mathrm{KO}$, et al. Preparation and characterization of polypropylene carbonate bio-filler (eggshell powder) composite films. Int J Polym Anal. 2014;19:637-47.

12. Tiimob BJ, Jeelani S, Rangari VK. Eggshell reinforced biocomposite-an advanced "green" alternative structural material. J Appl Polym Sci. 2016;133:1-10.

13. Rahman GS, Aftab H, Islam MS, Mukhlish MZB, Ali F. Enhanced physico-mechanical properties of polyester resin film using $\mathrm{CaCO}_{3}$ filler. Fibers Polym. 2016;17:59-65.

14. Shuhadah S, Supri AG. LDPE-isophthalic acid modified egg shell powder composites (LDPE/ESPI). J Physiol Sci. 2009;20:87-98.

15. Hashmi MSJ, Mridha S, Naher S. Mechanical properties modification of polyethylene (PE) for $\mathrm{CaCO}_{3}$ particulated composites. Adv Mat Res. 2011;264-265:880-7.

16. Toro P, Quijada R, Peralta R, Yazdani-Pedram M. Influence of grafted polypropylene on the mechanical properties of mineral-filled polypropylene composites. J Appl Polym Sci. 2007;103(4):2343-50.

17. Vincent SR, Jaafar M, Palaniandy S. Properties of calcium Carbonate/mica and calcium farbonate/talc filled polypropylene composites. J Eng Sci. 2014;10:41-7.

18. Teixeira SCS, Moreira MM, Lima AP, Santos LS, da Rocha BM, de Lima ES, et al. Composites of high density polyethylene and different grades of calcium carbonate: mechanical, rheological, thermal, and morphological properties. J Appl Polym Sci. 2006;101:2559-64.

19. Azman NAN, Islam MR, Parimalam M, Rashidi NM, Mupit M. Mechanical, structural, thermal and morphological properties of epoxy composites filled with chicken eggshell and inorganic CC particles. Polym Bull. 2019;77(2):805-21.

20. Khalaf N. Mechanical properties of filled high density polyethylene. J Saudi Chem Soc. 2015;19:88-91.

21. Wahab A, Habib M, Nawaz N. Preparation, characterization, and properties of polyethylene composites partially filled with calcium carbonate through co-rotating conical twin-screw extrusion. Int J Res Appl Sci Eng Technol. 2019;7(3):2401-4. 
22. Mohan TP, Kanny K. Thermal, mechanical and physical properties of nanoegg shell particle-filled epoxy nanocomposites. J Compos Mater. 2018;52(29):3989-4000.

23. Murugan S, Munusamy Y, Muniandy M, Ismail H. Development of HDPE-modified eggshell composite. Polym Compos. 2016;39(5):1630-7.

24. Owuamanam SI. Fabrication and characterization of bio-epoxy eggshell composites [MSc thesis]. Canada: University of Saskatchewan; 2019.

25. Ucurum M, Bayram O, Toraman OY, Kılıc H, Yalcın S. Changes of surface properties of calcite particles with calcium stearate using conventional experimental design and properties of coated calcite. Physicochem Probl Miner Proces. 2018;54(3):688-700.

26. Hassen AA, Dizbay-Onat M, Bansal D, Bayush T, Vaidya $\mathrm{U}$. Utilization of chicken eggshell waste as a bio-filler for thermoplastic polymers: thermal and mechanical characterization of polypropylene filled with naturally derived $\mathrm{CaCO}_{3}$. Polym Polymer Compos. 2015;23:653-62.

27. Rahmani M, Ashenai F, Payganeh G. Effect of surface modification of calcium carbonate nanoparticles on their dispersion in the polypropylene matrix using stearic acid. Mech Ind. 2014;67:63-7.

28. Mihajlović SR, Vučinić DR, Sekulić ŽT, Milićević SZ, Kolonja BM. Mechanism of stearic acid adsorption to calcite. Powder Technol. 2013;245:208-16.

29. Yoğurtcuoğlu E, Uçurum M. Surface modification of calcite by wet-stirred ball milling and its properties. Powder Technol. 2011;214:47-53.

30. Shah AH, Zhang Y, Xu X, Abdul Qadeer Dayo AQ, Li X, Wang $\mathrm{S}$, et al. Reinforcement of stearic acid treated egg shell particles in epoxy thermosets: structural, thermal, and mechanical characterization. Materials (Basel). 2018;11:1872-90.

31. Buakaew W, Yupaporn R, Suppakarn N, Sutapun W. Mechanical, thermal and morphological properties of poly(butylene succinate) filled with bio-functional filler from eggshell waste. Adv Mat Res. 2013;699:57-62.

32. Cao Z, Daly M, Clémence L, Geever LM, Major I, Higginbotham $\mathrm{CL}$, et al. Chemical surface modification of calcium carbonate particles with stearic acid using different treating methods. Appl Surf Sci. 2016;378:320-9.

33. Zeng Y-X, Zhong X-W, Liu Z-Q, Chen S, Li N. Preparation and enhancement of thermal conductivity of heat transfer oil-based MoS nanofluids. J Nanomater. 2013;4:1-6.

34. Shi X, Rosa R, Lazzeri A. On the coating of precipitated calcium carbonate with stearic acid in aqueous medium. Langmuir. 2010;26(11):8474-82.

35. Zaman HU, Beg MDH. Effect of CC contents on the properties of polyethylene nanocomposites sheets. Fibers Polym. 2014;15(4):839-46.

36. Bhagavatheswaran ES, Das A, Rastin H, Saeidi H, Jafari SH, Vahabi H, et al. The Taste of Waste: The edge of eggshell over calcium carbonate in acrylonitrile butadiene rubber. J Polym Environ. 2019;27:2478-89.

37. Lin J-H, Pan Y-J, Liu C-F, Huang C-L, Hsieh C-T, Chen C-K, et al. Preparation and compatibility evaluation of polypropylene/ high density polyethylene polyblends. Materials (Basel). 2015;8:8850-9.

38. Apone S, Bongiovanni R, Braglia M, Scalia D, Priola A. Effects of thermomechanical treatments on HDPE used for TLC ducts. Polym Test. 2003;22(3):275-80.

39. Basilia B, Panganiban MEG, Collado AAVC, Pesigan MOD. Yro PAd. Study on the functionality of nano-precipitated calcium carbonate as filler in thermoplastics. J Solid Mech Mater Eng. 2007;1(4):564-70.

40. Bartczak Z, Argon AS, Cohen RE, Weinberg M. Toughness mechanism in semi-crystalline polymer blends: II. high-density polyethylene toughenedwith calcium carbonate filler particles. Polymer (Guildf). 1999;40:2347-65.

41. Lazzeri A, Zebarjad SM, Pracella M, Cavalier K, Rosa R. Filler toughening of plastics. Part I-The effect of surface interactions on physico-mechanical properties and rheological behavior of ultrafine $\mathrm{CaCO}_{3} / \mathrm{HDPE}$ nanocomposites. Polymer (Guildf). 2005;46:827-44.

42. Saeedi M, Sharahi SJ. Morphological and thermal properties of HDPE/CaCO3 nanocomposites: effect of content of nano and MFI. In: International Conference on Nanotechnology and Biosensors IPCBEE 25@. Singapore: IACSIT Press; 2011. p. 34-38.

43. Ertürk AT, Şahin T. Degradation effect of different environments on characteristics of calcium carbonate reinforced polyethylene. Gazi Univ J Sci. 2017;30(4):489-500.

44. Dombrowski T, Hrizuk SA, Dizikes LJ. Method for improved melt flow rate of filled polymeric resin. United States patent WO2006012505A1 2006.

45. Abbas-Abadi MS, Haghighi MN, Yeganeh H. Effect of the melt flow index and melt flow rate on the thermal degradation kinetics of commercial polyolefins. J Appl Polym Sci. 2012;126:1739-45.

46. Sahai RSN, Patel S, Santwani S, Jaiswal R. Studies on mechanical properties of calcium carbonate filled PPO composite with coupling agent. Int J Res Eng Technol. 2017;7(6):129-41.

47. Jang K-S. Mineral filler effect on the mechanics and flame retardancy of polycarbonate composites: talc and kaoline. Polymers (Basel). 2016;16:379-86.

48. Adeosun SO, Usman MA, Akpan EI, Dibie WI. Characterization of LDPE reinforced with calcium carbonate-fly ash hybrid filler. J Miner Mater Charact Eng. 2014;2:334-45.

49. Fu Y-S, Feng X-Q, Laukec B, Mai Y-W. Effects of particle size, particle/matrix interface adhesion and particle loading on mechanical properties of particulate-polymer composites. Compos, Part B Eng. 2008;39:933-61.

50. Hayeemasae N, Lim WS, Hanafi I. Sustainable use of eggshell powder in the composite based on recycled polystyrene and virgin polystyrene mixture. Int J Polym Anal Ch. 2019;24:266-75.

51. Melo PMA, Macêdo OB, Barbosa GP, Ueki MM, Silva LB. High-density polyethylene/mollusk shell-waste composites: effects of particle size and coupling agent on morphology, mechanical and thermal properties. J. Mater. Res. Technol. 2019;8(2):1915-25.

52. Buakaew W. Preparation of High Density Polyethylene and Poly(Butylene Succinate) filled with bio-fillers from Eggshell [thesis]. Tailand: Suranaree University of Technology; 2013.

53. Bonse BC, Molina LM. Effect of calcium carbonate particle size and content on polyamide 6 processing and properties. AIP Conf Proc. 2016

54. Hassan SB, Aigbodion VS, Patrick SN. Development of polyester/eggshell particulate composites. Tribology in Industry. 2012;34(4):217-25.

55. Somdee P, Hasook A. Effect of modified eggshell powder on physical properties of poly(lactic acid) and natural rubber composites. Materials Today: Proceedings. 2017;4:6502-11.

56. Murugan S, Munusamy Y, Ismail H. Effects of chicken eggshell filler size on the processing, mechanical and thermal properties of PVC matrix composite. Plast Rubber Compos. 2017;46:42-51. 